ACCepted for publication in Astrophysical Journal LetTers

Preprint typeset using $\mathrm{LAT}_{\mathrm{E}} \mathrm{X}$ style emulateapj v. 12/16/11

\title{
AN EXTREME GRAVITATIONALLY REDSHIFTED IRON LINE AT 4.8 KEV IN MRK 876
}

\author{
Eugenio Bottacini $^{1}$, Elena Orlando ${ }^{1}$, Jochen Greiner ${ }^{2}$, Marco Ajello $^{3}$, Igor Moskalenko ${ }^{1}$, Massimo Persic ${ }^{4,5}$ \\ Accepted for publication in Astrophysical Journal Letters
}

\begin{abstract}
$\mathrm{X}$-ray spectral lines at unforeseen energies are important because they can shed light on the extreme physical conditions of the environment around the supermassive black holes of active galactic nuclei (AGN). Mrk 876 displays such a line at $4.80_{-0.04}^{+0.05}$ rest-frame energy. A possible interpretation of its origin can be found in the hotspot scenario. In this scenario the primary radiation from a flare in the hot corona of an AGN illuminates a limited portion of the accretion disk that emits by fluorescence. In this context the line can represent an extreme gravitationally redshifted $\mathrm{Fe}$ line originating on the accretion disk below 6 gravitational radii from a rotating supermassive black hole. The correct estimate of the line significance requires a dedicated approach. Based on an existing rigorous approach, we have performed extensive Monte Carlo simulations. We determine that the line is a real feature at $\sim 99 \%$ confidence level.

Subject headings: accretion, accretion disks — galaxies: active — galaxies: individual (Mrk 876) line: formation — line: identification — X-rays: galaxies
\end{abstract}

\section{INTRODUCTION}

Active galactic nuclei (AGN) are generous emitters of X-rays. These photons are thought to be produced in the central region of the AGN. There, optical/UV photons from the accretion disk are inverse-Comptonized by electrons (hot corona) thereby producing the primary $\mathrm{X}$-ray radiation seen by an observer as a powerlaw spectrum. This primary radiation also irradiates the accretion disk that reflects it through several reprocessing steps (e.g. Matt et al. 1991) including fluorescence emission of $\mathrm{K} \alpha$ lines of most abundant elements (George \& Fabian 1991). Therefore, emission lines from reflection spectra are good diagnostics of the environment of the inner region of AGN (Ross \& Fabian 1993; Fabian et al. 2000, and references therein). Among emission lines from abundant elements in AGN spectra, the most common is the $\mathrm{Fe} \mathrm{K} \alpha$ line at $6.4 \mathrm{keV}$.

Even more intriguing are transient fluorescence emission lines. In this case the primary radiation can originate in magnetic field reconnection (Merloni \& Fabian 2001) dissipating the energy input into the Comptonizing corona and it irradiates a limited portion (hotspot) of the accretion disk for a short time. As a result the emerging fluorescence lines are transient. They are also affected by Doppler and gravitational effects causing distorted line shapes and photon energy shifts (Fabian et al. 2000). However, shifted line energies can be caused also by AGN outflows and inflows. These shifts to unexpected energies led to conflicting interpretations in the literature (Vaughan \& Uttlev 2008) that are comprehensively discussed in Turner et al. (2010). Therefore, any new study

\footnotetext{
eugenio.bottacini@stanford.edu

${ }^{1}$ W.W. Hansen Experimental Physics Laboratory \& Kavli Institute for Particle Astrophysics and Cosmology, Stanford University, USA

2 Max-Planck-Institut für extraterrestrische Physik, Giessenbachstrasse 1, D-85748 Garching, Germany

${ }^{3}$ Department of Physics and Astronomy, Clemson University, Clemson SC 29634-0978, USA

${ }^{4}$ INAF-Trieste, via G.B.Tiepolo 11, I-34143 Trieste, Italy

${ }^{5}$ INFN-Trieste, via A.Valerio 2, I-34127 Trieste, Italy
}

is important.

In this Letter, we report on a detection in a Swift/XRT observation of an emission line at $4.8 \mathrm{keV}$ rest-frame energy of the optically discovered Seyfert type 1 AGN Mrk 876. We study the significance of the line with Monte Carlo simulations. We then analyze all other available $\mathrm{X}$-ray observations of the source (by GINGA, $X M M-N e w t o n$, and Swift/XRT) to constrain the physical origin of the line.

\section{LINE FEATURE IN $S W I F T / X R T$ SPECTRUM OF MRK 876}

Mrk 876 is part of an optically selected sample of the Palomar-Green (PG) Bright Quasar Survey sample (Schmidt \& Green 1983). The redshift of the host galaxy is $z=0.1385$ (Lavaux \& Hudson 2011) corresponding to $551.4 \mathrm{Mpc}$ for $H_{0}=73 \mathrm{~km} \mathrm{~s}^{-1} \mathrm{Mpc}^{-1}$ assuming Hubble flow. The Galactic absorption toward Mrk 876, assuming solar abundance, has been measured by Elvis et al. (1989) using NRAO $140 \mathrm{ft}$ telescope of Green Bank finding a value of $\mathrm{N}_{H}^{\text {gal }}=2.66 \times 10^{20}$ atoms cm ${ }^{-2}$ with $5 \%$ error. The source was observed by Swift/XRT (obs id: 00050300004) on the 2005-03-01. For the analyses of the observation we used HEAsoft 6.11 .1 and xrtproducts. Events for the spectral analysis are extracted from a circular region of interest centered on the source position having a radius of $\sim 20$ pixels (corresponding to $\sim 47$ arcsec, Moretti et al. 2004). The background is extracted from a nearby source-free circular region of interest of 50 pixel radius. The best fit model for the backgroundsubtracted source spectrum is obtained by adding to the power-law a Gaussian-component at 4.22 that models well the residuals (see Figure 11). All the parameters of the model are free to float obtaining a line energy of $4.22_{-0.04}^{+0.05} \mathrm{keV}$ and a width of $90_{-75}^{+76} \mathrm{eV}$ compatible with the energy resolution of $\sim 100 \mathrm{eV}$. This leads to a fit result of $\chi^{2}=133.64 / 132$ d.o.f. The F-test shows strong evidence that the improved fit result is not due by chance. To verify that the line is not due to the background, we analyze the background spectrum. It does not display any line feature and it accounts for a mere $2.3 \%$ of the 
total net count rate $1.409 \times 10^{-1}$ count s $^{-1}$. Furthermore its contribution to the line flux is negligible. The absence of spectral features and the low level of background flux exclude any artifact that could give origin to the line. This is not surprising as Swift/XRT is known for its low background level due to its low orbit especially for energies above $2 \mathrm{keV}$ (Moretti et al. 2007). In Section 2.1 we will estimate more properly the probability for the Gaussian component to be a real spectral feature not caused by statistical fluctuations.

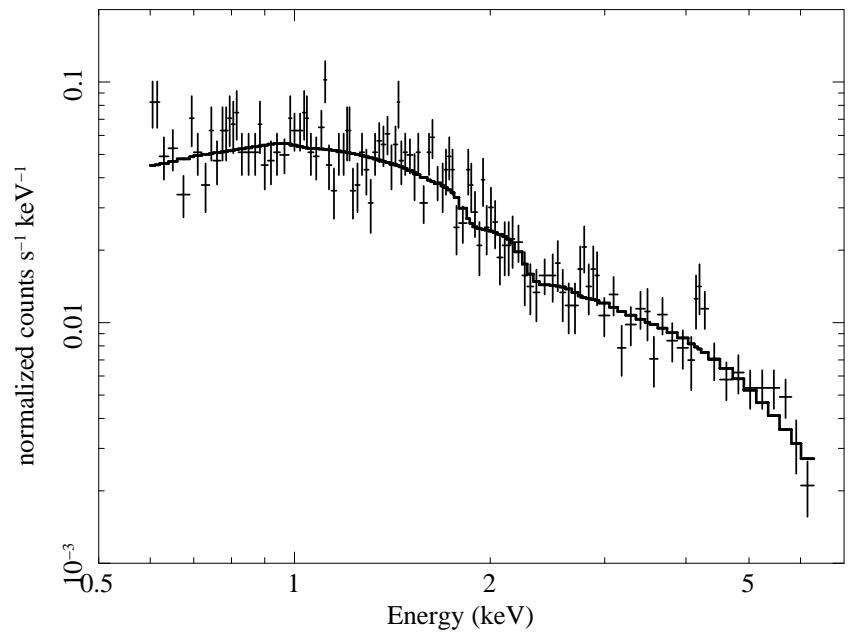

Fig. 1.- Swift/XRT spectrum of Mrk 876 (observer's frame). Excess at $4.22 \mathrm{keV}$.

\subsection{Statistical significance of the line feature at 4.22 ke $V$ observed-frame energy}

There is general consensus that line searches justified by observational data must be in need of validation through randomized trials (Protassov et al. 2002). Turner et al. (2010) used such an approach that we adopt with further improvements.

To estimate the significance of the line at $4.22 \mathrm{keV}$ observed-frame energy, we carry out Monte Carlo simulations in the wide energy range of $0.6-6.5 \mathrm{keV}$. Our null hypothesis is: the spectrum as measured by Swift/XRT is a power-law with absorption fixed to the galactic value (null model). This spectrum is simulated by using the XSPEC command fakeit accounting for the same exposure and instrument response files of the observation. The simulated spectral data are grouped to a minimum of 15 counts per energy bin as done for our measured spectrum thereby accounting for adequate statistics. This is iterated $10^{4}$ times. Each single simulated spectrum is fitted with our null model. The results are used to simulate a further spectrum as before. This procedure accounts for the uncertainties of the null hypothesis model (Markowitz et al. 2006; Tombesi et al. 2010). Again the obtained simulated spectrum is fitted with the null model thereby obtaining the $\chi_{n u l l}^{2}$. The same spectrum is then fitted with the null model and an additional new Gaussian-line component throughout the $3.5-6.5 \mathrm{keV}$ energy range according to the work of Porquet et al. (2004b) and successfully applied by Markowitz et al. (2006) and Turner et al. (2010). This energy range is stepped through by energy bins whose size corresponds to the spectral energy resolution of Swift/XRT at each given energy (Short et al. 2002). The line energy is allowed to freely float within this energy bin, similarly to what is done in a sophisticated line search by Turner et al. (2010). Starting from an initial value of zero the line normalization freely varies between positive and negative values. The line width is free starting from initial value of $\sigma=0$ to a maximum value corresponding to the energy resolution. For each simulated spectrum the best chi square $\left(\chi_{\text {best }}^{2}\right)$ is used to obtain the maximum $\Delta \chi^{2}\left(=\chi_{\text {best }}^{2}-\chi_{\text {null }}^{2}\right)$. Consequently, the distribution of the $10^{4} \Delta \chi^{2}$ indicates the fraction of lines caused by chance fluctuations whenever the value of the $\Delta \chi^{2}$ exceeds the threshold value $\left(\Delta \chi_{t h r}^{2}=8.77\right)$.

To account for the low statistic domain we have performed further $10^{4}$ simulations as described above but applying Churazov-weighting (Churazov et al. 1996). The $\Delta \chi^{2}$ need to exceed the $\Delta \chi_{t h r}^{2}=8.59$.

However, 15 counts bin $^{-1}$ might be low to confidentially use chi-square statistic as the probability distribution might be Poisson (Cash 1979). Therefore, to more confidentially estimate the line significance, we run again $10^{4}$ simulations but applying $C$-statistic (Cash 1979) based on maximum-likelihood ratio method. Even though $C$ statistic is suited for unbinned data, we group spectra to 1 count $\mathrm{bin}^{-1}$ avoiding zero-count bins as $C$ statistic is best performed by XSPEC for minimum binning (Teng et al. 2005). In this case $\Delta C\left(=C_{b e s t}-C_{n u l l}\right)$ must exceed $\Delta C_{t h r}=10.95$.

As a result we find that thresholds of each of our 3 approaches chi-square, chi-square Churazov-weighted, and $C$-statistic in each of their $10^{4}$ spectra are exceeded by $1.36 \%, 1.50 \%$, and $1.21 \%$, respectively. These similar results are not surprising as differences between $\chi^{2}$ and $C$-statistic are expected when number of counts bin ${ }^{-1}$ and number of bins are low (Nousek \& Shue 1989), while here the latter is large. Thus, the null hypothesis, of the measured spectrum being described by a power-law and galactic absorption, is rejected by each of the 3 approaches with a probability of roughly 99\%. Figure 2 displays the cumulative (blue) and the frequency histograms (red) of the $\Delta C$ distribution: at $\sim 10.95$ only a small fraction contributes to the total number of trials.

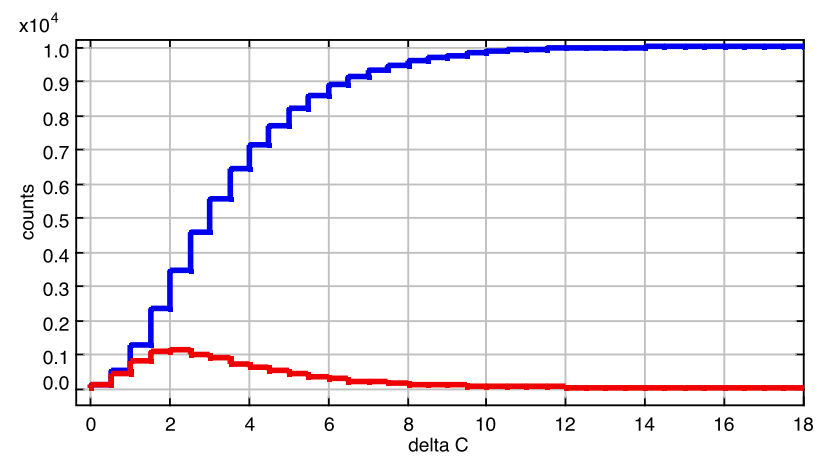

Fig. 2.- Cumulative (blue) and frequency (red) histograms of delta C-statistic. 


\subsection{Testing for systematic effects in the spectrum}

To further investigate on the reality of the line we undertake several tests. We extract spectra from different positions on the sky (R.A., decl. $=(243.3390,65.6440)$, $(243.2566,65.5802),(243.4108,65.7710))$ without detecting any spectral signature at $4.22 \mathrm{keV}$. This excludes systematics in this specific observation. This is supported also by the fact that there has been no report on instrumental line features at $4.22 \mathrm{keV}$ due to calibration residuals of the effective area of Swift/XRT (Godet et al. 2009). A further test is to extract the spectrum from Mrk 876 from nested and smaller circles of the source. The line feature at $4.22 \mathrm{keV}$ observed-frame energy still persists. We further verify that the line feature is not due to an astronomical transient feature not associated to Mrk 876. For this purpose, we first extract a light curve of Mrk 876 in the $4.0-4.4 \mathrm{keV}$ energy range from the observation displaying the line (see Figure 4). We find that the flux is stable over the entire observation time. Therefore, a possible transient feature among our analyzed Swift/XRT observations not associated to Mrk 876 in the image would be visible. Thus, we compare images in the $4.0-4.4 \mathrm{keV}$ energy band including the one with the line feature (see Figure 3). There is no evidence for transient features. These tests reinforce the idea that the line at $4.22 \mathrm{keV}$ is not instrumental and that it is associated to Mrk 876.

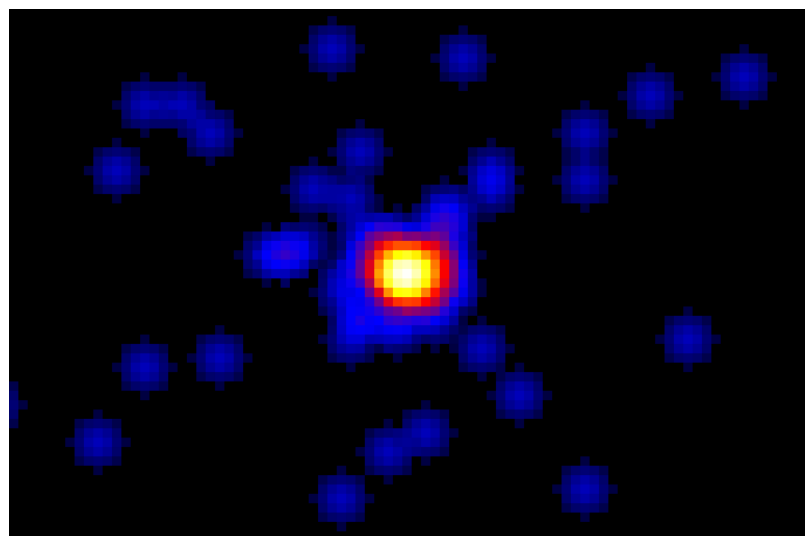

FIG. 3.- Swift/XRT observation (00050300004) 4.0-4.4 keV.

\subsection{Further $X$-ray observations}

Mrk 876 was targeted by Swift/XRT with further 16 observations (see Table 1). We neglect the 2 having less than $0.6 \mathrm{ksec}$ exposure time and we analyze them in the same way as described earlier. We find that the remaining spectra are best described by a power-law model with absorption fixed to the Galactic value.

We have reanalyzed the GINGA (Makino 1987) spectrum of Mrk 876 as published in Lawson \& Turner (1997). The 2 to $18 \mathrm{keV}$ spectrum is best fitted with a power-law and absorption parameter fixed to the Galactic value (see Table [1).

$X M M-N e w t o n$ has targeted the source twice (Table 1). These observations were analyzed in Piconcelli et al. $(2005)$ and in Porquet et al. (2004a). We have processed $X M M-N e w t o n$ Observation Data Files using the XMMNewton Scientific Analysis Software (Gabriel et al. 2004) version 10.0. Source spectra are extracted from a circular region with radius of 30 arcsec while background spectra are extracted using a nearby source-free region having 60 arcsec radius. The background-subtracted spectra are best fitted with a broken power-law and absorption fixed to the Galactic value (see Table 1). There is no evidence for any line feature including the Fe $\mathrm{K} \alpha$ line at $6.4 \mathrm{keV}$.

Spectra were analyzed with XSPEC 12 (Arnaud 1996).

\section{DISCUSSIONS}

The importance of $\mathrm{X}$-ray spectral lines at unforeseen energies is their capability to shed light on the extreme physical conditions where they originate. Any new detection is important in light also of future missions with improved throughput and energy resolution (e.g. Athena+; Nandra et al. 2013).

\subsection{The line feature in Mrk 876}

Being a Seyfert type 1 AGN, Mrk 876 allows for clean view on the innermost accretion region. As such, it is an ideal target for a case study. None of the GINGA, Swift/XRT, and XMM-Newton observations reveal the narrow $\mathrm{Fe} \mathrm{K} \alpha$ line at $6.4 \mathrm{keV}$. This is in agreement with previous analyses of GINGA observation (Lawson \& Turner 1997) and XMM-Newton observations (Porquet et al. 2004a; Piconcelli et al. 2005). This line, often seen in AGN, is interpreted as Fe fluorescence from cold (neutral) matter far from the inner accretion disk (Yaqoob \& Padmanabhan 2004; Page et al. 2004). A viable explanation for its absence in Mrk 876 can come from the X-ray Baldwin effect (Iwasawa \& Taniguchi 1993), an anti-correlation between the equivalent width of the Fe $\mathrm{K}$ line and $\mathrm{X}$-ray luminosity, which can be explained by the drop of the covering factor of the molecular AGN torus (Page et al. 2004). Therefore, it is not surprising that Mrk 876 does not display a steady Fe $\mathrm{K} \alpha$ line being one of the most luminous AGN among the systematically studied Palomar-Green (PG) Bright Quasar Survey sample at $\mathrm{X}$-rays (Porquet et al. 2004a). However, for one Swift/XRT observation (obs id: 00050300004) we find a significant line emission at a confidence level of $99 \%$ at $4.80_{-0.04}^{+0.05} \mathrm{keV}$ rest-frame energy. At the same rest-frame energy a similar line but with lower confidence level has been reported by Petrucci et al. (2007) for Mrk 841 that is a Seyfert type 1 AGN.

\subsection{On the origin of the $4.8 \mathrm{keV}$ rest-frame line}

In the following we consider the possible origin of our transient line in the accretion disk hotspot scenario (Nayakshin \& Kazanas 2001; Turner et al. 2002). The inclination angle of Mrk 876 's accretion disk of $15.4_{-6.8}^{+12.1}$ deg is independently constrained by Bian \& Zhao (2002). This very low inclination angle leads to the gravitational redshift dominance of the line over the Doppler effect in both rotating (Kerr) black hole (Laor 1991) and a non-rotating (Schwarzschild) black hole environments (Fabian et al. 1989). As a result the line profile tends to a single peak (Fabian et al. 1989), which is intrinsically very narrow (<100 eV; Navakshin \& Kazanas 2001) and mainly subject to gravitational redshift. The observed gravitational redshift factor is $\nu_{\text {observed }} / \nu_{\text {rest frame }}$ 
$=0.75_{-0.01}^{+0.01}$ where errors account for the line energy uncertainty. Following Fabian et al. (1989) this factor cannot be obtained by a non-rotating black hole. We constrain the emission-region on the accretion disk by simulating the model of Laor (1991) accounting for its different emissivity power-law indices and taking care of the uncertainties of the inclination angle of the source. The gravitational redshift factor of $0.75_{-0.01}^{+0.01}$ cannot be obtained from emission-regions at radii above $6 r_{g}$ (where $\left.r_{g}=G M_{B H} / c^{2}\right)$ reaching at most a factor of 0.89 . On the contrary for radii below $6 r_{g}$ such a gravitational redshift factor can be well reproduced. Only accretion disks of Kerr black holes can extend below $6 r_{g}$ (Thorne 1974) emphasizing once again the environment of a rotating black hole. On the other hand, for Schwarzschild black holes, a detailed line profile study by Reynolds \& Begelman (1997) allows for the fluorescence $\mathrm{Fe} \mathrm{K} \alpha$ line emission from material spiraling toward the event horizon even below $6 r_{g}$. However, the profile is predicted to be double-peaked for accretion disk inclination angles other than face-on, in contrast to our finding. As a result, the line can be reproduced by a rotating black hole only. This is in agreement with a range of spectral parameters predicted in an accurate study by Dovčiak et al. (2004) for a black hole with angular momentum $a=0.9$ and disk inclination $\theta_{0}=30 \mathrm{deg}$ for a hotspot on an accretion disk emitting photons from its last stable orbit $r_{m s}+d r$. Unfortunately the low signal-to-noise spectrum does not allow for a more constraining fit with the Laor-model or with the more complex kerrdisk-model. The same low signal-to-noise ratio prevents from attempting a cross-correlation study between the line light curve and the continuum light curve (both shown in Figure 4) that are predicted to correlate in the hotspot framework (Dovčiak et al. 2004). Also correlation studies between Swift/XRT and Swift/BAT are impossible due to the low signal-to-noise observations of the latter (Baumgartner et al. 2013). Unfortunately, no UVOT data were taken, which could constrain the geometry of the emitting region (George \& Fabian 1991). On the other hand the independent estimates of the black hole mass of Mrk 876 (Kaspi et al. 2000; Bian \& Zhao 2002) allow us to compute the orbital period for the hotspot on the accretion disk. Dovčiak et al. (2004) predict the line to appear for a relatively short fraction of the total orbital period of the hotspot at the distance from the black hole where the line occurs. Thus, we estimate the orbital period using the formula below of Bardeen et al. (1972) as performed in Dovčiak et al. (2004) where $r$ is in units of gravitational radii.

$$
P=310\left(r^{\frac{3}{2}}+a\right) \frac{M_{B H}}{10^{7} M_{\odot}} \quad[\mathrm{s}] .
$$

We assume the photons emitted from a spinning black hole $a=0.9$ at a disk radius of $6 r_{g}$. Taking the two independently inferred black hole masses of Mrk $876 M_{B H}=1.3 \times 10^{9} \mathrm{M}_{\odot}$ (Bian \& Zhao 2002) and $M_{B H}=2.4 \times 10^{8} \mathrm{M}_{\odot}$ (Kaspi et al. 2000), the resulting orbital periods are $\mathrm{P}=628 \mathrm{ksec}$ and $\mathrm{P}=135 \mathrm{ksec}$, respectively. Both are significantly longer than the Swift/XRT observation time $(\sim 25 \mathrm{ksec})$. This is in agreement with the narrow lines to appear for a fraction of the total period as the flare dies out or emission gets below detectability (Dovčiak et al. 2004). The Swift/XRT observation (obs id: 00050302001) taken 6 days later without any line detection, makes the hotspot scenario a consistent picture. Also the line feature has never been observed in any other $\mathrm{X}$-ray observation confirming the transient nature of the event that reinforces the hotspot origin.

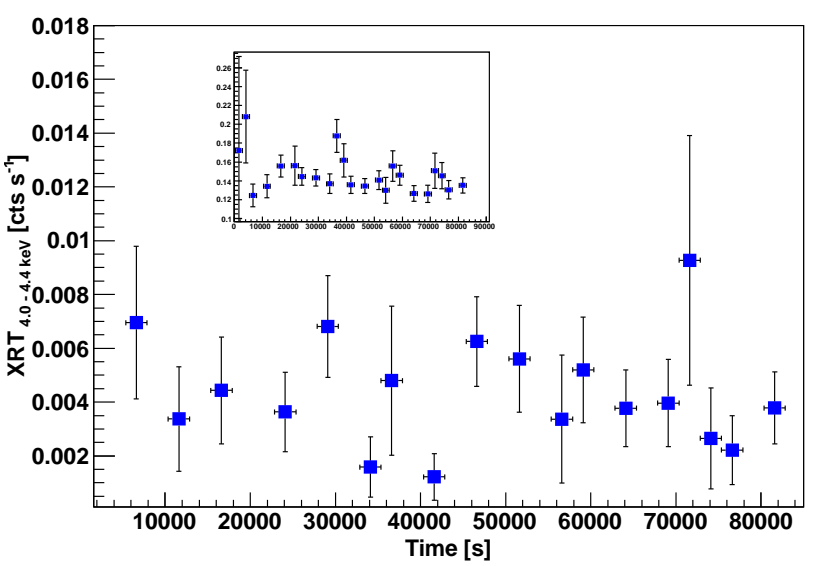

FIG. 4. - Light curve of observation 00050300004 in the energy band $4.0-4.4 \mathrm{keV}$ and $0.6-6.5 \mathrm{keV}$ (inset).

Another hypothetical origin of the line could be the emission from enhanced abundances of sub-Fe elements resulting from Fe spallation. In this scenario, Fe nuclei hit by cosmic-rays are converted into elements of atomic number $Z$ below Fe. The element emitting closest to our line energy would be Vanadium at $4.9 \mathrm{keV}$ (rest-frame energy). However, the absence of the Fe $\mathrm{K} \alpha$ at $6.4 \mathrm{keV}$, which would still be the strongest line in this scenario (Skibo 1997), makes it an unlikely explanation. Also the transient nature of the line would be difficult to explain.

In a further hypothesis a $\sim 4.7 \mathrm{keV}$ (rest-frame energy) spectral feature could originate also from Compton down-scattered Fe line from FeXXV at $6.7 \mathrm{keV}$ (Fabian et al. 2012). However, the resulting gaussian component is expected to be much broader $(\sim 0.4 \mathrm{keV})$. Also, this component needed to be present in all our analyzed Mrk 876 spectra. Thus, the down-scattered Fe line is also very unlikely.

A redshifted Fe line could originate also from inflows and outflows of material (not related to the accretion disk) in the vicinity of the black hole. In this region the material would be subject to strong gravitational effects reaching mildly relativistic $(\sim 0.4 \mathrm{c})$ velocities (Tombesi et al. 2010) causing an energy shift of emission lines. This is the interpretation for a line shift observed in Mrk 766 in a detailed and time-resolved study by Turner et al. (2004) in presence of a strong $\mathrm{Fe} \mathrm{K} \alpha$ component at 6.4 $\mathrm{keV}$. In fact, the absence of this latter component in all our analyzed observations of Mrk 876 disfavors this interpretation since it is difficult to imagine one single blob of material emitting an Fe line. In addition our observations a month earlier (obs id: 00050300005) and 6 days later (obs id: 00050302001) with Swift/XRT should have detected the corresponding $6.4 \mathrm{keV}$ line. 


\subsection{On the significance of the line}

A detailed study by Vaughan \& Uttlev (2008) suggests that reported line significances might be altered by a publication bias where only most significant among detected spectral features are mentioned. Such an important issue can be addressed by a statistical study of a sample as a whole where several lower significance lines are detected as e.g. in Tombesi et al. (2010). Instead, our study reports a case study of a transient event in the hotspot scenario. Therefore, to determine the detection likelihood we cannot incorporate the non-presence of the lines in other observations. Also is it here impossible to address the publication bias as it must address the bias for a source population at large (Vaughan \& Uttley 2008).

\section{CONCLUSIONS}

Our analysis of the Swift/XRT observation of Mrk 876 (obs id: 00050300004) has revealed an emission line at $4.80_{-0.04}^{+0.05} \mathrm{keV}$ rest-frame energy associated to the source. The line is detected at $\sim 99 \%$ confidence level through Monte Carlo simulations using $\chi^{2}$ and $C$-statistic. The possible origin of the line is the hotspot scenario in which a flare in the hot corona (possibly due to magnetic reconnection) illuminates a limited portion of the accretion disk that emits the Fe line by fluorescence. Due to the independent constraints of the low inclination of Mrk 876's accretion disk (15.4 deg) and the observed gravitational redshift, we can constrain that the line is emitted from the accretion disk at radii below $6 r_{g}$ (where $\left.r_{g}=G M_{B H} / c^{2}\right)$ from a Kerr black hole. The alternative possible origins due to Fe spallation, down-scattered Fe line, and mass inflows/outflows are disfavored in light of the absence of the $6.4 \mathrm{keV}$ Fe line and the variability studies due to the many available observations of Swift/XRT, XMM-Newton, and GINGA.

To the best of our knowledge, this is the first time that a transient line revealed at $99 \%$ confidence level is unambiguously associated to the hotspot scenario.

Considerable improvement of the Letter is due to robust comments of the anonymous referee. E.B. acknowledges NASA grant NNX13AF13G. The authors thank Giacomo Vianello for helpful discussions and the instrument teams for the observations.

Facilities: Swift, XMM, Ginga 


\section{REFERENCES}

Arnaud, K. A. 1996, in Astronomical Society of the Pacific Conference Series, Vol. 101, Astronomical Data Analysis Software and Systems V, ed. G. H. Jacoby \& J. Barnes, 17

Bardeen, J. M., Press, W. H., \& Teukolsky, S. A. 1972, ApJ, 178, 347

Baumgartner, W. H., Tueller, J., Markwardt, C. B., Skinner, G. K., Barthelmy, S., Mushotzky, R. F., Evans, P. A., \& Gehrels, N. 2013, ApJS, 207, 19

Bian, W., \& Zhao, Y. 2002, A\&A, 395, 465

Cash, W. 1979, ApJ, 228, 939

Churazov, E., Gilfanov, M., Forman, W., \& Jones, C. 1996, ApJ, 471,673

Dovčiak, M., Bianchi, S., Guainazzi, M., Karas, V., \& Matt, G. 2004, MNRAS, 350, 745

Elvis, M., Wilkes, B. J., \& Lockman, F. J. 1989, AJ, 97, 777

Fabian, A. C., Iwasawa, K., Reynolds, C. S., \& Young, A. J. 2000, PASP, 112, 1145

Fabian, A. C., Rees, M. J., Stella, L., \& White, N. E. 1989, MNRAS, 238, 729

Fabian, A. C. et al. 2012, MNRAS, 419, 116

Gabriel, C. et al. 2004, in Astronomical Society of the Pacific Conference Series, Vol. 314, Astronomical Data Analysis Software and Systems (ADASS) XIII, ed. F. Ochsenbein, M. G. Allen, \& D. Egret, 759

George, I. M., \& Fabian, A. C. 1991, MNRAS, 249, 352

Godet, O. et al. 2009, A\&A, 494, 775

Iwasawa, K., \& Taniguchi, Y. 1993, ApJ, 413, L15

Kaspi, S., Smith, P. S., Netzer, H., Maoz, D., Jannuzi, B. T., \& Giveon, U. 2000, ApJ, 533, 631

Laor, A. 1991, ApJ, 376, 90

Lavaux, G., \& Hudson, M. J. 2011, MNRAS, 416, 2840

Lawson, A. J., \& Turner, M. J. L. 1997, MNRAS, 288, 920

Makino, F. 1987, Astrophys. Lett., 25, 223

Markowitz, A., Reeves, J. N., \& Braito, V. 2006, ApJ, 646, 783

Matt, G., Perola, G. C., \& Piro, L. 1991, A\&A, 247, 25

Merloni, A., \& Fabian, A. C. 2001, MNRAS, 328, 958
Moretti, A et al. 2004, in Society of Photo-Optical Instrumentation Engineers (SPIE) Conference Series, Vol. 5165, X-Ray and Gamma-Ray Instrumentation for Astronomy XIII,

ed. K. A. Flanagan \& O. H. W. Siegmund, 232-240

Moretti, A. et al. 2007, in Society of Photo-Optical Instrumentation Engineers (SPIE) Conference Series, Vol. 6688, Society of Photo-Optical Instrumentation Engineers (SPIE) Conference Series

Nandra, K., Barret, D., Barcons, X., et al. 2013, arXiv:1306.2307

Nayakshin, S., \& Kazanas, D. 2001, ApJ, 553, L141

Nousek, J. A., \& Shue, D. R. 1989, ApJ, 342, 1207

Page, K. L., O’Brien, P. T., Reeves, J. N., \& Turner, M. J. L. 2004, MNRAS, 347, 316

Petrucci, P. O. et al. 2007, A\&A, 470, 889

Piconcelli, E., Jimenez-Bailón, E., Guainazzi, M., Schartel, N., Rodríguez-Pascual, P. M., \& Santos-Lleó, M. 2005, A\&A, 432, 15

Porquet, D., Reeves, J. N., O’Brien, P., \& Brinkmann, W. 2004a, A\&A, 422, 85

Porquet, D., Reeves, J. N., Uttley, P., \& Turner, T. J. 2004b, A\&A, 427, 101

Protassov, R., van Dyk, D. A., Connors, A., Kashyap, V. L., \& Siemiginowska, A. 2002, ApJ, 571, 545

Reynolds, C. S., \& Begelman, M. C. 1997, ApJ, 488, 109

Ross, R. R., \& Fabian, A. C. 1993, MNRAS, 261, 74

Schmidt, M., \& Green, R. F. 1983, ApJ, 269, 352

Short, A. D., Ambrosi, R. M., \& Turner, M. J. L. 2002, Nuclear Instruments and Methods in Physics Research A, 484, 211

Skibo, J. G. 1997, ApJ, 478, 522

Teng, S. H., Wilson, A. S., Veilleux, S., Young, A. J., Sanders,

D. B., \& Nagar, N. M. 2005, ApJ, 633, 664

Thorne, K. S. 1974, ApJ, 191, 507

Tombesi, F., Cappi, M., Reeves, J. N., Palumbo, G. G. C.,

Yaqoob, T., Braito, V., \& Dadina, M. 2010, A\&A, 521, A57

Turner, T. J., Kraemer, S. B., \& Reeves, J. N. 2004, ApJ, 603, 62

Turner, T. J., Miller, L., Reeves, J. N., Lobban, A., Braito, V., Kraemer, S. B., \& Crenshaw, D. M. 2010, ApJ, 712, 209

Turner, T. J. et al. 2002, ApJ, 574, L123

Vaughan, S., \& Uttley, P. 2008, MNRAS, 390, 421

Yaqoob, T., \& Padmanabhan, U. 2004, ApJ, 604, 63 
TABLE 1

MRK 876 SOFt X-RAY SPECTRAL Fit. ${ }^{*}$ OBSERVATION DiSPLAYing THE LiNe.

\begin{tabular}{|c|c|c|c|c|c|c|c|c|c|}
\hline $\begin{array}{l}\text { instrument } \\
{[\text { obs } i d]}\end{array}$ & $\begin{array}{l}\text { start } \\
{[\text { date }]}\end{array}$ & $\begin{array}{l}\text { expo } \\
{[\text { sec }]}\end{array}$ & $\Gamma$ & $\Gamma_{\text {hard }}$ & $\begin{array}{c}\mathrm{E}_{\text {break }} \\
{[\mathrm{keV}]}\end{array}$ & $\begin{array}{c}\text { norm } \\
{\left[10^{-3} \mathrm{ph} \mathrm{keV}^{-1} \mathrm{~cm}^{-2}\right]}\end{array}$ & Chi-square & d.o.f. & $\begin{array}{c}\text { flux } 2-6 \mathrm{keV} \\
{\left[10^{-12} \mathrm{erg} \mathrm{cm}^{-2} \mathrm{~s}^{-1}\right]}\end{array}$ \\
\hline GINGA & 1991-05-11 12:10:00 & 112208 & $1.47_{-0.11}^{+0.10}$ & $\ldots$ & $\ldots$ & $0.8_{-0.1}^{+0.2}$ & 12.44 & 11 & $2.97_{-0.27}^{+0.25}$ \\
\hline XMM 0102040601 & 2001-04-13 19:38:25 & 12825 & $2.50_{-0.15}^{+0.13}$ & $1.81_{-0.22}^{+0.12}$ & $0.97_{-0.19}^{+0.45}$ & $1.01_{-0.10}^{-0.1}$ & 149.77 & 136 & $2.43_{-0.17}^{+0.20}$ \\
\hline XMM 0102041301 & 2001-08-29 07:45:05 & 7919 & $2.48_{-0.07}^{+0.06}$ & $1.76_{-0.14}^{+0.15}$ & $1.46_{-0.34}^{+0.24}$ & $1.85_{-0.10}^{+0.07}$ & 184.10 & 200 & $3.30_{-0.13}^{+0.18}$ \\
\hline XRT 00050300001 & $2004-12-23 \quad 00: 12: 23$ & 3774 & $1.12_{-0.16}^{+0.17}$ & $\ldots$ & $\ldots$ & $\begin{array}{r}0.59_{-0.09}^{+0.09} \\
\end{array}$ & 14.08 & 12 & $3.20_{-0.34}^{+0.32}$ \\
\hline XRT 00050300005 & 2005-01-26 00:37:41 & 12669 & $1.26_{-0.07}^{+0.06}$ & $\ldots$ & $\ldots$ & $0.94_{-0.05}^{+0.06}$ & 117.45 & 105 & $4.28_{-0.20}^{+0.18}$ \\
\hline XRT 00050300004* & 2005-03-01 23:59:01 & 25458 & $1.50_{-0.05}^{+0.06}$ & $\ldots$ & $\ldots$ & $0.77_{-0.04}^{+0.03}$ & 142.41 & 135 & $2.52_{-0.10}^{+0.09}$ \\
\hline XRT 00050302001 & 2005-03-08 02:08:54 & 23158 & $1.36_{-0.05}^{+0.05}$ & $\ldots$ & $\ldots$ & $0.87_{-0.04}^{+0.04}$ & 169.76 & 153 & $3.42_{-0.12}^{+0.10}$ \\
\hline XRT 00050302002 & 2005-03-09 00:33:52 & 9400 & $\begin{array}{r}1.37_{-0.08}^{+0.05} \\
\end{array}$ & $\ldots$ & $\ldots$ & $0.87_{-0.07}^{+0.06}$ & 72.19 & 69 & $3.37_{-0.18}^{+0.12}$ \\
\hline XRT 00050303001 & 2005-03-09 08:55:52 & 7725 & $\begin{array}{r}1.27_{-0.09}^{+0.10} \\
\end{array}$ & $\ldots$ & $\ldots$ & $0.83_{-0.07}^{+0.07}$ & 49.32 & 53 & $3.67_{-0.23}^{+0.23}$ \\
\hline XRT 00050303003 & 2005-03-12 00:50:03 & 3879 & $1.35_{-0.12}^{+0.12}$ & $\ldots$ & $\ldots$ & $0.92_{-0.11}^{+0.07}$ & 23.71 & 31 & $3.67_{-0.32}^{+0.29}$ \\
\hline XRT 00050303004 & 2005-03-24 07:07:51 & 10269 & $1.42_{-0.08}^{+0.08}$ & $\ldots$ & $\ldots$ & $0.86_{-0.07}^{+0.05}$ & 94.27 & 71 & $3.12_{-0.17}^{+0.18}$ \\
\hline XRT 00035308001 & 2006-05-30 07:31:01 & 8463 & $1.76_{-0.09}^{+0.08}$ & $\ldots$ & $\ldots$ & $\begin{array}{l}-0.07 \\
1.41_{-0.09}^{+0.08}\end{array}$ & 76.01 & 77 & $3.32_{-0.18}^{+0.17}$ \\
\hline XRT 00035308002 & 2006-06-18 01:12:01 & 4422 & $1.77_{-0.14}^{+0.14}$ & $\ldots$ & $\ldots$ & $1.25_{-0.14}^{+0.09}$ & 40.36 & 30 & $2.90_{-0.26}^{+0.23}$ \\
\hline XRT 00035308003 & 2007-09-25 00:11:00 & 2214 & $1.83_{-0.18}^{+0.19}$ & $\ldots$ & $\ldots$ & $1.28_{-0.15}^{+0.17}$ & 4.94 & 12 & $2.78_{-0.31}^{+0.36}$ \\
\hline XRT 00035308004 & 2007-10-02 17:02:00 & 2590 & $1.69_{-0.14}^{+0.15}$ & $\ldots$ & $\cdots$ & $1.16_{-0.14}^{+0.12}$ & 17.16 & 21 & $2.97_{-0.30}^{+0.28}$ \\
\hline XRT 00035308005 & 2007-10-04 04:16:00 & 2887 & $1.96_{-0.16}^{+0.16}$ & $\ldots$ & $\ldots$ & $1.63_{-0.16}^{+0.14}$ & 11.58 & 19 & $3.01_{-0.27}^{+0.30}$ \\
\hline XRT 00035308006 & 2007-10-04 10:35:00 & 5927 & $1.81_{-0.10}^{+0.11}$ & $\ldots$ & $\ldots$ & $1.37_{-0.10}^{+0.11}$ & 41.06 & 51 & $3.03_{-0.21}^{+0.27}$ \\
\hline XRT 00091642002 & 2013-10-02 13:21:59 & 1012 & $1.66_{-0.34}^{+0.35}$ & $\ldots$ & $\ldots$ & $2.13_{-0.46}^{+0.45}$ & 7.97 & 10 & $5.64_{-0.94}^{+0.21}$ \\
\hline
\end{tabular}

